\title{
L'économie minière de l'orpaillage artisanal dans les sociétés post-conflit : jeux des acteurs et enjeux de développement et de coopération internationale. Étude de cas en Côte d'Ivoire
}

\author{
Constant Soko ${ }^{\mathrm{a}}$
}

RÉSUMÉ. L'économie minière de l'orpaillage artisanal est apparue en Côte d'Ivoire dans les années 2000, à la suite d'une rébellion qui s'est emparée du nord du pays. Les rebelles ont mis en place cette économie en vue de trouver des ressources afin de faire face à l'effort de guerre et d'apporter un semblant d'activité économique dans les différentes régions sous leur contrôle. Depuis, cette activité a continué et est devenue l'activité économique principale dans les différentes zones rurales du pays après la réunification du pays. Cette économie, bien qu'apportant des revenus conséquents aux mineurs et aux populations locales, ne peut pas assurer, à elle seule, le développement local et a besoin d'un soutien aussi bien de l'État que des multinationales d'extraction minière pour un partenariat public privé. Ceci est d'autant plus important que les mineurs artisanaux ont de l'expertise aussi bien sur le plan de l'extraction que celui de la commercialisation. Ils maitrisent le marché local et sous régional qui est différent de celui des multinationales. Une coopération entre l'État, les orpailleurs artisanaux et les multinationales d'extraction minière pourrait permettre de créer de milliers d'emplois et réduire les conséquences environnementales et les risques d'accident, ce qui pourrait entrainer un véritable développement local dans les différentes régions. La coopération internationale devrait en effet se pencher sur ces derniers aspects.

\begin{abstract}
$\boldsymbol{A B S T R A C T}$. Traditional gold mining economy appeared in Côte d'Ivoire in the early 2000s following the rebellion where the northern part of the country was taken over. The rebels set up this economy to find resources to finance the war effort and seemingly create economic activity in the various regions under their control. Since then, this activity has continued to be and became the main economic activity in the various rural areas of the country after the reunification of the country. This economy, while providing significant income to miners and local populations, cannot by itself ensure local development and needs support from both the state and the mineral mining multinationals for public-private partnership. This is more so important since the traditional miners have expertise in both extraction and marketing. They master the local and subregional market, that is different from the multinational markets. Cooperation between the state, traditional gold miners and mining multinationals could create thousands of jobs and reduce environmental consequences and accident risks. This could lead to real local development in the various regions and is where international co-operation should take action.
\end{abstract}

\section{Problématique}

Depuis des siècles, l'Afrique de l'Ouest est l'une des régions productrices d'or les plus connues dans le monde. Les richesses de Mansa Musa, l'empereur du
Mali au XIVe siècle, étaient si considérables qu'il a tristement ruiné le marché de l'or du Moyen-Orient et de l'Afrique du Nord pendant une décennie, après avoir charitablement distribué de l'or tout au long de son pèlerinage à La Mecque, par voie terrestre, en 1324.

a Université Felix Houphouët-Boigny d'Abidjan, UFR Sciences de l'Homme et de la Société 
Depuis les années 1990, nous assistons à un développement important du secteur minier en Afrique de l'Ouest sous l'impulsion d'une part, de politiques minières nationales attractives et d'autre part, d'un fort investissement du secteur privé étranger. Ce développement, voulu par les pays et souvent encadré par les institutions internationales telles que la Banque Mondiale, a permis l'ouverture d'un nombre relativement important d'exploitations minières et se traduit par un poids significatif dans le produit intérieur brut (PIB) et les recettes d'exportation de chaque pays. Aujourd'hui, la région demeure une importante source d'or. L'exploitation minière est en forte progression, entrainant ainsi une croissance économique remarquable (environ $5 \%$ ) dans la région. Les pays comme le Ghana font partie des 10 principaux producteurs industriels du monde, tout comme la Côte d'Ivoire, le Mali et le Burkina Faso qui se classent parmi les 15 plus importants producteurs africains.

En Côte d'Ivoire, le secteur de l'or a affiché une croissance au cours des dernières années, les exportations industrielles étant passées de 4,2 tonnes en 2008 à 10,4 tonnes en 20131. Le pays reste toutefois un producteur moyen, comparativement au Ghana qui dispose, à quelques différences près, des mêmes réserves que la Côte d'Ivoire. La production annuelle de la Côte d'Ivoire est estimée à 40 tonnes contre 76 tonnes pour le Ghana. En 2010, la Côte d'Ivoire était le vingtième producteur aurifère au monde et le cinquième en ordre d'importance sur le continent africain après l'Afrique du Sud, le Ghana, le Mali et le Burkina Faso. Dans la même année de 2010, 10 \% des exportations ivoiriennes étaient générées par l'or. En réalité, les deux principales entreprises multinationales (Equigold et Perseus) engagées dans l'exploitation minière se partagent la manne aurifère ivoirienne : elles ont le monopole des actions sur les mines les plus productives. En 2015, la production aurifère a connu une hausse de $15 \%$ par rapport à 2014, avec 23,5 tonnes d'or extrait. En 2015, classée au $7 \mathrm{e}$ rang africain et $42^{\mathrm{e}}$ mondial des pays producteurs d'or, la Côte d'Ivoire évalue la contribution de sa production à hauteur de $5 \%$ du PIB, avec une production annuelle de 23,5 tonnes.

Il faut rappeler que l'exploitation de l'or n'est pas récente en Côte d'Ivoire. Pendant longtemps, la Société des Mines Internationales (SMI), exploitant la mine d'or d'Ity à Zouhan-Hounien dans la région de Danané2, a été le flambeau de l'exploitation aurifère en Côte d'Ivoire. Aujourd'hui, grâce à une série de politiques favorables aux investisseurs, d'autres sociétés d'exploitation minière de l'or telles que Randgold (Jamasmie, 2014), dont le PDG croit que le pays est le nouvel eldorado de l'or et qu'il pourrait même dépasser un jour les résultats du Ghana, se sont établies (Ford, 2014). C’est également le cas d'autres mines telle que la mine d'or de Bonikro $^{3}$ dans le département de Hiré4, exploitée par la société minière Equigold, qui y a investi 50 milliards et celle de Tongon (village situé au nord de la Côte d'Ivoire dans la sous-préfecture de Tengrela $)^{5}$, qui appartient à la société Perseus, propriété du groupe sud-africain Randgold Ressources, qui lui-même fait partie du consortium Rio Tinto. Cette dernière est considérée comme la plus grande mine d'or de Côte d'Ivoire avec en réserve huit millions d'onces (environ 120 tonnes d'or). Avec un investissement prévu de 200 milliards de FCFA, la mine d'or a produit son premier lingot de 9,5 kg, présenté au gouvernement le 8 novembre 2005. Il existe également une mine d'or dans le village d'Angovia dans la souspréfecture d'Aboisso, dont l'exploitation a été interrompue par la société Cogema du fait de la crise post-électorale de 2010. Par ailleurs, la société Etruscan Côte d'Ivoire, filiale de la société canadienne Etruscan Ressources Inc., qui devait exploiter la mine d'or du village d'Agbaou dans la sous-préfecture de Dabou ${ }^{6}$ à partir de fin 2010, a vu son activité compromise à cause de la situation de crise post-électorale.

La problématique minière révèle que, très souvent, les pays producteurs et leurs populations ne profitent pas des dividendes miniers. C'est le cas de la Côte d'Ivoire où l'important potentiel minier ne profitant pas aux populations, notamment dans les zones d'exploitation, ces dernières se sont lancées dans des activités d'orpaillage clandestin qui ne cesse de prendre de l'ampleur. En juillet 2017, le gouvernement avait annoncé la fermeture de 429 sites d'orpaillage illicites dans le pays.

En effet, l'exploitation artisanale de l'or n'a réellement débuté sérieusement qu'au cours de la guerre civile qui a éclaté en 2003. Durant la crise de près d'une décennie qui s'est ensuivie la moitié nord du pays échappait au contrôle du gouvernement central à Abidjan, ce qui laissait l'exploitation et le commerce de l'or entièrement dans la sphère illicite 
et aux mains des Forces nouvelles, le principal groupe rebelle. Depuis la fin des hostilités, la production artisanale s'est fortement intensifiée dans le pays. De l'avis de certains experts, l'exploitation illégale serait nettement supérieure à celle faite dans les conditions légales. On extrait de l'or de façon artisanale dans 24 des 31 régions de la Côte d'Tvoire. Cette activité concerne plus de 500000 mineurs d'or artisanal selon les chiffres du ministère de l'Industrie et des Mines de la Côte d'Ivoire7. Le 20 juillet 2016 au cours d'une cérémonie dédiée à l'évaluation de son programme de lutte, le gouvernement a annoncé que quelque 429 sites d'orpaillage illicite ont été abandonnés, fermés et 66 orpailleurs clandestins, interpellés. La plupart des activités se déroulent aux alentours de Bouaké et de Katiola, où le ministère de l'Industrie et des Mines a identifié 28 zones d'exploitation. Les experts évaluent à « environ 30 tonnes d'or par an la production artisanale qui échappe à l'État » privant ainsi ce dernier "d'importantes recettes" (ONUCI, 2010). Le ministère des Mines lui-même affirmait en 2015 que «l'orpaillage clandestin a pris des dimensions inquiétantes, durant les dix dernières années, faisant perdre à l'État quelque 479,22 milliards de FCFA soit environ 818 millions \$ US ». Ainsi, pour le gouvernement, la lutte contre l'orpaillage clandestin est d'abord un moyen d'optimiser son secteur minier en augmentant son apport en termes de PIB et en consolidant les investissements porteurs après la levée- en avril 2014- de l'embargo sur le minerai imposé par les Nations Unies durant la crise militaro-politique (Conseil de sécurité des Nations Unies, 2014). En plus des pertes financières, l'État ivoirien considère l'orpaillage clandestin comme une source potentielle d'accroissement des conflits fonciers et de la criminalité. L'orpaillage artisanal semble donc s'être bien incrusté dans le paysage économique des zones rurales, supplantant à maints endroits l'économie agricole jugée moins lucrative. Cependant, le développement de cette activité économique soulève de nombreux problèmes dont celui des droits de l'homme et de la dégradation de l'environnement et des ressources naturelles.

L'orpaillage ou l'exploitation artisanale des exploitations minières est-il «l'enfant pauvre » de la coopération internationale dans le domaine minier? Quelle est l'origine de l'orpaillage artisanal? Comment cette activité est-elle arrivée en Côte d'Ivoire? Comment naissent et se développent ces activités d'orpaillage?
Quelles sont les perceptions locales liées à cette activité? Quels en sont les acteurs et leurs rôles? Quels sont les impacts économiques de l'orpaillage artisanal (différents types d'emplois et d'activités économiques générés, les gains financiers perçus par chaque acteur)? Quel est l'impact de l'orpaillage sur les équilibres sociaux et communautaires, sur la santé, sur la sécurité, sur l'éducation et sur l'économie agricole et locale? Quel est l'impact de l'orpaillage artisanal sur l'environnement?

\section{Objectifs}

Cette étude a pour objectif de mettre en lumière les jeux des acteurs et les enjeux de développement économique, social, environnemental et de coopération internationale dans le domaine de l'économie minière de l'orpaillage artisanal en Côte d'Ivoire. Plus spécifiquement, il s'agit de retracer l'origine de cette activité dans le pays, d'identifier les différents acteurs, mais aussi d'analyser:

- les perceptions locales liées à cette activité;

- les caractéristiques sociodémographiques des orpailleurs;

- les résultats économiques de l'orpaillage artisanal;

- l'impact social et communautaire;

- l'impact environnemental;

- les risques liés à l'activité d'orpaillage artisanal.

\section{Méthodologie}

Sur le plan méthodologique, nous allons dans un premier temps construire l'échantillon. Dans un deuxième temps, nous allons présenter les outils de collecte des données et dans un troisième temps, nous allons montrer comment nous avons collecté les données.

\section{1 Échantillonnage (constitution des bases de sondage et tirages des échantillons)}

Au titre de l'échantillonnage pour la collecte des données, nous avons adopté une approche par stratification selon les catégories d'acteurs: propriétaires de puits, orpailleurs de type alluvionnaire, broyeurs et concasseurs. Cet échantillonnage a été effectué pour tous les sites de production, afin de mieux cerner les spécificités des différentes localités concernées par l'activité d’orpaillage. En outre, 
à l'intérieur de chaque strate, un tirage aléatoire simple a été appliqué pour former un échantillon composé de $15 \%$ des effectifs de chaque catégorie d'acteurs. La procédure proprement dite est définie comme suit: (i) une sélection de tous les sites de production d'orpaillage; (2) un tirage de $15 \%$ dans chaque site selon les types d'acteurs existants (propriétaires de puits, orpailleurs alluvionnaires, broyeurs et concasseurs).

\subsection{Matériel et outils utilisés}

Pour mener à bien cette étude, nous avons eu recours à certains outils de travail dont : (i) des guides d'entretien élaborés par nos soins; (ii) des cartes topographiques, (iii) un appareil numérique pour effectuer des prises de vue; (iv) des logiciels tels que Arcview, etc.; (v) un ordinateur portable et une clé USB pour la saisie, l'enregistrement et l'analyse des données; (vi) un véhicule pour les besoins de déplacement sur le terrain en vue de la collecte des données.

\section{3 Échantillonnage et élaboration des instruments de collecte}

Dans le cadre de la collecte de données, nous avons utilisé trois types de questionnaires et un guide d'entretien selon la population cible concernée :

- un questionnaire concernant les propriétaires/responsables de puits et les orpailleurs s'activant dans l'alluvionnaire (tranchées) qui a servi à collecter les informations portant sur les caractéristiques sociodémographiques des membres ou ouvriers des puits, le degré de production d'or de chaque puits, les biens d'équipement, le circuit de commercialisation, la répartition des revenus, etc.;

- un questionnaire administré aux individus évoluant dans l'orpaillage alluvionnaire. Il a servi à collecter les informations relatives à la production d'or, aux équipements utilisés et les principaux clients (acheteurs de l'or);

- un questionnaire adressé aux chefs des unités de l'activité de broyage/ concassage. Ce questionnaire a permis de collecter les informations relatives aux caractéristiques sociodémographiques, au service fourni par les broyeurs/concasseurs, aux équipements utilisés et aux charges et dépenses;
- un guide d'entretien pour l'animation des focus groups. Les personnes ciblées étaient composées de commerçants, des concasseurs, des broyeurs, des restaurateurs, etc. dont les activités sont impactées par l'orpaillage. Cet outil a permis de collecter des informations relatives à la perception des acteurs sur: (i) les impacts économiques de l'orpaillage; (ii) les impacts sur la santé de la population; (iii) les impacts sur l'agriculture; (iv) les impacts sur l'environnement, etc.

\subsection{Collecte des données}

La collecte a été faite en utilisant une méthode CAPI (Computer Assisted Personal Interview Collecte de données assistée par ordinateur). Le recueil des informations repose essentiellement sur les dires d'experts et les échanges avec les acteurs locaux riverains des mines (exploitants agricoles et maraîchers, collectivités territoriales, populations, chefs traditionnels et coutumiers), au travers des entretiens de compréhension, l'exploitation des données secondaires et des visites de terrain. Pour ce faire, plusieurs instruments de collecte de données, capables de répondre aux objectifs assignés ont été utilisés en vue de recueillir les informations sur le terrain. Il s'agit de l'observation directe et de l'entretien de compréhension. Ces entretiens qui sont au nombre de 25 nous ont permis de mieux cerner la problématique, d'identifier les conflits et les pollutions et/ou rumeurs de pollution des activités minières sur les ressources en eau qui ont eu lieu au niveau des six mines en exploitation en Côte d'Ivoire, mais aussi et surtout de recueillir les préoccupations des populations locales. Ils ont permis également d'analyser le jeu des acteurs autour des mines et d'apprécier le degré de mise en œuvre des textes et lois relatifs à l'exploitation minière, l'utilisation durable des ressources en eau et la protection de l'environnement. Un choix raisonné a été effectué en fonction des objectifs fixés pour l'identification de la catégorie de personnes à rencontrer dans le cadre de cette étude exploratoire.

Les deux visites de terrain, effectuées dans la zone minière de Hiré (située dans le centre ouest du pays), de Tortiya (situé dans le nord du pays) et à Bouna (dans l'est du pays) nous ont permis, quant à elles, au travers des observations directes, de décrire l'environnement biophysique (espaces agricoles et maraîchers, types et nature des sols, etc.) et de repérer la situation de la 
mine par rapport aux ressources en eau à proximité (cours d'eau, barrages hydrauliques, puits et forages, etc.).

\subsection{Focus group}

Au cours des focus groups, nous avons utilisé une approche par strate. Pour ce qui est des groupes, le type d'échantillonnage utilisé est l'échantillonnage aléatoire stratifié. À la suite, dans chaque strate, un sondage aléatoire simple a été fait pour la sélection des répondants en vue de constituer des groupes de discussion composés chacun de commerçants, ouvriers et acteurs évoluant dans l'activité d'orpaillage. Chaque groupe est composé de 10 personnes. Cette approche permet de connaitre la perception des populations relative à l'impact de l'exploitation artisanale de l'or sur l'éducation des enfants, la santé de la communauté, l'évolution des mœurs, l'agriculture, la vie économique, la qualité de l'eau, l'environnement, etc.

\subsection{Traitement et analyse des données}

Au titre du traitement des données, deux logiciels ont été utilisés : le logiciel (CSPRO 7) pour l'application de collecte et le logiciel (STATA 15) pour la correction des valeurs aberrantes et manquantes ainsi que les différentes tabulations. Après chaque journée de collecte, les données rassemblées ont fait l'objet de vérifications.

\section{Résultats}

\subsection{La rébellion, les chefs de guerre et la naissance de l'activité en Côte d'Ivoire}

L'expansion de l'orpaillage est relativement récente dans la plupart des zones rurales du pays. Elle a débuté à partir de 2002. C'est la crise militaropolitique de 2002 à 2010 qui en a favorisé la naissance. Les premières exploitations ont été le fait d'orpailleurs venus du Burkina Faso (notamment de la région aurifère de Gawa) et du Mali. Ces derniers ont été installés dans les zones sous contrôle de la rébellion avec l'appui de chefs de guerre de la rébellion ivoirienne qui ont gouverné cette partie du territoire entre 2002 et 2010. Ainsi, en Côte d'Ivoire, un officier supérieur, le lieutenant-colonel Issiaka Ouattara, a bénéficié suffisamment de revenus de mines d'or illégales pour mobiliser un groupe de 500 éléments armés qui, en constituant une force parallèle à l'armée, menaçait la stabilité et la sécurité de la nation (Hunter, 2017). Selon les enquêteurs des Nations Unies, quelque $15800 \mathrm{mi}-$ neurs informels étaient actifs sur le site de la mine de Gamina, contrôlée par Ouattara, où étaient produites environ 2,5 tonnes d'or par an; un or vendu bien en deçà de sa valeur (Conseil de Sécurité des Nations Unies 2014). On voit donc des liens entre l'exploitation aurifère illégale et les guerres. Ce fut le cas dans les activités de l'est de la République Démocratique du Congo, pour lesquelles des données sont disponibles (ONUDC 2011) ${ }^{8}$.

À la fin de la rébellion, le phénomène a connu une nouvelle vitalité, favorisée par une hausse vertigineuse du prix de l'or, qui a atteint jusqu'à 1600 \$ US l'once (31,10 grammes) en 2011 contre 250 dollars quelques années plus tôt. L'activité s'est ainsi avérée, dans un contexte local de forte pauvreté, comme une opportunité économique bien plus lucrative que l'économie agricole traditionnelle dans les différentes zones rurales. «Le cacao nous a donné un peu d'argent, mais les choses ont changé. Ce que tu gagnais en deux ans, si tu as la chance, tu as ça en une semaine avec l'or! » a assuré un jeune exploitant du village d'Agbahou dans la sous-préfecture de Hiré.

\subsection{Les perceptions des orpailleurs et de leurs activités économiques}

\subsubsection{Don, contre don et réciprocités entre les mineurs et l'or!}

Les différentes croyances associées à l'extraction de l'or sont envisagées ici à la fois comme une stratégie de gestion du risque et comme les facettes d'un idiome du changement qui empruntent des références locales et extérieures. Comme telles, elles voient dans l'activité minière et ses dangers un véritable défi. Souvent, les risques et les périls liés à l'extraction sont perçus comme un phénomène inévitable - car l'or et la richesse dépendent de forces (ou d'esprits) exigeant une « récompense »même si, bien sûr, les mineurs veulent avoir la chance de leur côté et un travail sans accident. Y réussir dépend d'un large spectre de pratiques religieuses et parareligieuses : sacrifices, consultation de prêtres, cérémonies spéciales, divination, acquisition d'objets magiques ou d'amulettes (vendus en grand nombre sur les marchés locaux). Dans les 
régions des mines d'or, les prêtres, les devins et les marabouts, ainsi que différentes sectes nordaméricaines sont aujourd'hui présents, attirés par la demande des communautés de mineurs et de ces nouveaux marchés.

Pour rendre compte de la nature risquée de l'extraction de l'or, de ses succès ou de ses échecs, de ses accidents ou de sa chance, on mobilise la réciprocité qui existerait entre l'or - souvent vu comme un être vivant - ou ses esprits et les mineurs. Si la montagne et ses temples ne sont pas respectés et que de l'or est extrait, quelque chose doit être donné en retour. "La montagne demande du sang » est une expression courante pour dire que la montagne requiert des cérémonies et est responsable d'accidents graves. Il existe même une croyance selon laquelle on ne peut échapper aux accidents mortels et que ceux-ci garantissent le succès final de l'entreprise. Comme Schneider (1990) le dit à propos des Lobi du Burkina Faso, des superstitions similaires à celles sur l'or se rencontrent dans d'autres zones d'Afrique de l'Ouest. Elles sont bien sûr très fluides, et l'adhésion à ces croyances varie d'un individu à l'autre suivant la formation religieuse reçue. En ce sens, mon analyse diffère de celle de Nash (1993) qui étudia les mineurs d'étain en Bolivie et leurs relations avec les esprits de la montagne.

\subsection{2 Éthique religieuse entre le mineur et l'or}

Des membres de l'Église pentecôtiste nouvellement créée dans l'un des villages voisins parlent de la « volonté de Dieu » et de la chance comme du résultat de la prédétermination de tout individu et des forces divines. Quelques devins expliquent la présence de l'or par le travail des ancêtres; d'autres le nient purement et simplement. L'interprétation, omniprésente dans d'autres contextes africains, selon laquelle le succès ou la malchance seraient les produits de sources occultes de pouvoir n'est pas consensuelle parmi les mineurs. Le travail de Taussig (1980) sur les mineurs colombiens soulève la même critique: il essentialise des tendances au sein de communautés conçues comme des ensembles unifiés, et néglige les négociations intenses et les différenciations existant selon les sous-cultures de ces communautés. Même s'il se concentre sur la marchandisation et le changement social, Taussig affirme de façon trop aiguë une divergence entre logiques et moralités de production et de consommation qui n'est pas valable pour tous les mineurs d'or. Il existe une grande diversité d'interprétations et de croyances locales, qui ont des origines extrêmement variées et sont liées à des processus divergents de communication.

\subsubsection{Perception des creuseurs}

Les processus identitaires des jeunes creuseurs se voient dans leurs pratiques sociales sous-jacentes et les discours qui ont contribué à leur émergence. La particularité de l'identité émergente du creuseur provient d'une combinaison spécifique d'attitudes de travail et de consommation, d'une situation de migrant, de pressions externes et d'une forte conscience de soi. Si, malgré les nombreux défis et risques, l'extraction de l'or lui parait profitable, cela est dû à une absence d'alternatives pour gagner de l'argent ou accumuler du capital. Les creuseurs d'or se réinventent eux-mêmes comme mineurs. Ils répondent à leur situation particulière de migrants en créant des normes et des standards de comportement spécifiques. Ils font preuve d'une flexibilité remarquable et d'une créativité sémantique, une façon pour eux de s'approprier une situation incertaine et de modifier constamment les conditions sociales et politiques de leur environnement. Ils agissent ainsi malgré (ou à cause de) leurs nombreux conflits internes, les défis et les risques qui dominent leur vie quotidienne.

\subsubsection{L'orpaillage perçu comme un processus d'initiation et l'acquisition d'une nouvelle identité}

Majoritairement jeunes, les nouveaux mineurs sont soumis à une double initiation: d'une part, une initiation dans le monde socioprofessionnel des mineurs, un groupe masculin disposant d'un style de vie distinct, d'une éthique de travail et de comportement; de l'autre, une initiation qui correspond à un cycle complet de migration. C'est donc une identité supplémentaire que les mineurs acquièrent, pertinente d'abord pour le monde de la mine, principalement liée à cette activité particulière et non à un groupe ethnique. Cette identité dérive aussi largement de la situation de jeunes migrants partageant les mêmes aspirations et ne se confond pas avec d'autres identités dépendantes des régions dont ils sont originaires. 


\subsection{5 Être mineur : une stratégie de débrouille}

Tous ces jeunes migrants ne viennent pas sur les sites miniers pour devenir des mineurs professionnels, et ne désirent pas, dans leur majorité, passer leur vie dans un tel univers. Personne ne naît creuseur d'or. C'est avant tout une stratégie économique et sociale de débrouille, dans la mesure où la migration de travail fait partie du quotidien de l'Afrique de l'Ouest depuis plus de dix ans. La plupart des mineurs espèrent quitter un jour ces luttes quotidiennes et vivre ailleurs une existence plus plaisante. Il s'agit donc d'un double processus d'adaptation aux circonstances, qui souligne leur distinction comme groupe: par l'adoption d'un style de vie et par la production de sens dans une situation incertaine.

\subsection{Les diffrérents acteurs de l'économie minière de l'orpaillage artisanal}

\subsubsection{Le pouvoir coutumier : chef de terre, chef de village et chef de canton, la notabilité et le comité de gestion}

La persistance de cette activité face à la volonté de l'État ivoirien de l'endiguer ne s'explique pas uniquement par les avantages économiques qu'elle génère. L'encastrement même de l'activité dans les structures sociales et les réseaux de pouvoir sur le plan local éclaire également les raisons de cette résistance.

Ce sont davantage les structures locales de pouvoir qui en ont garanti la persistance malgré les récurrentes mesures étatiques d'interdiction et de répression. Dans la plupart des localités du pays où se fait l'exploitation artisanale de l'or, la structure politique traditionnelle fait apparaitre trois figures influentes dans le système de gouvernance intégrant plusieurs villages : le chef de canton, le chef de terre et les chefs de village. Le chef de village, trônant à la tête d'une notabilité composée des chefs des lignages du village, assure l'administration quotidienne du village (interface entre le village et l'administration publique; gestion des conflits; etc.). Si le chef canton règne sur plusieurs villages en jouant le rôle de gestionnaire de conflit en dernier recours et de représentant politique de la tribu pour ce qui est de l'administration étatique, c'est le chef des terres de la tribu qui détient de loin l'autorité politique la plus influente dans l'organisation communautaire. La source de son pouvoir est d'ordre religieux et économique : il est l'adorateur principal des divinités de la terre, assisté d'adjoints-chefs de terre placés sous son autorité et repartis dans l'ensemble des villages de la tribu à laquelle appartient le village. Aucune initiative économique (agricole, pastorale et aurifère) sur les terres de la tribu ne peut prospérer sans l'assentiment du chef suprême de terre dont l'accord est marqué par la célébration d'une cérémonie d'adoration des génies pour obtenir leurs faveurs.

Dans le cas des activités aurifères, ce préalable cérémoniel est d'autant plus décisif qu'il répond aux croyances locales associées au métal précieux et à son exploitation. Selon ces croyances, l'or a avant tout une valeur spirituelle. L'anthropologue ivoirien Niangoran-Bouah traduit bien dans une étude, les conceptions idéologiques associées à l'or en pays akan et qui sont aussi largement partagées au sein de la plupart des communautés ouestafricaines : « [...] l'or, métal se trouvant à l'état pur dans la nature, n'est pas une créature simple, il est le métal des métaux, il est le plus noble, car inaltérable et éternel. L'or possède un esprit fort et redoutable ». Il ajoute que, toujours selon ces conceptions, « être vivant, l'or ne demeure pas en place dans la nature, il se déplace d'un point à un autre de la terre. Il peut se rendre visible et invisible» (Niangoran-Bouah, 1978, p.17).

La conséquence majeure découlant de ces croyances est que toute exploitation de l'or est préalablement soumise à des rites visant deux objectifs. D'une part, il s'agit d'apaiser cet «esprit fort et redoutable» au risque de subir une sanction s'exprimant sous forme de maladie incurable. D'autre part, ces rites permettent de garantir une quête fructueuse de ce métal «vivant» se «déplaçant d'un point à un autre de la terre ». Les libations, offrandes et prières officiées par le chef de terre en l'honneur des divinités de la terre, visent ainsi à faciliter l'exploitation du métal précieux et mystérieux. Ce rôle religieux du chef de terre le place ainsi au cœur même du dispositif du système d'exploitation de l'or.

Dans la plupart des villages, c'est le chef des terres qui donne son accord et officie toutes les cérémo- 
nies religieuses avant l'ouverture des sites d'exploitation artisanale du minerai. En échange de son intervention, une ristourne est prélevée sur les activités de chaque orpailleur et lui est reversée. Le chef de canton, figure importante de la tribu au niveau administratif, bénéficie également de la redistribution de la rente prélevée. L'important rôle du chef de terre s'exerce en étroite collaboration avec le chef de village qui intervient plus directement dans la régulation des activités d'exploitation se déroulant sur son territoire. Ce dernier est à la tête de la notabilité composée des chefs des clans dans le village.

Deux structures interviennent activement dans la régulation des activités d'orpaillage sur le plan villageois : la notabilité et un comité de gestion exclusivement dirigé par l'association des jeunes du village. La notabilité, organe politique du village, prend les décisions visant l'organisation de l'exploitation aurifère et la gestion des ressources qui en découlent. Elle décide de l'ouverture des zones d'exploitation en collaboration avec le chef de terre et décide de l'installation dans la zone d'orpailleurs rompus à la profession. La notabilité a en collaboration avec le comité de gestion fixé le coût des permis d'exploitation à 100000 FCFA (soit environ 171 \$ US) par exploitant. La rente recueillie serait en partie investie dans la construction d'infrastructures et autres dépenses communautaires (école, santé, etc.). Outre le fait que les jeunes autochtones sont exemptés de permis d'exploitation, une règle définie à leur avantage précise que tout exploitant étranger se doit d'employer en priorité les jeunes du village comme ouvriers. Le fruit de l'exploitation est partagé à parts égales entre l'exploitant en chef et son ouvrier. En tant qu'organe opérationnel, le comité de gestion veille à l'application des décisions de la notabilité. Il procède à la comptabilité des exploitations d'or ainsi que de leurs usagers. Il délivre les permis d'exploitation après achat, organise l'installation des migrants et assure le contrôle sécuritaire sur les sites. Un droit d'accès de 500 FCFA (soit environ $0,85 \$$ US) est quotidiennement encaissé auprès de tous les usagers comme droit d'entrée sur les sites. Le comité joue également le rôle de gestionnaire des éventuels conflits pouvant naitre entre exploitants.

L'expansion et la vitalité de l'orpaillage illicite dans plusieurs zones rurales de la Côte d'Ivoire, en dépit des mesures d'interdiction et de régulation adoptées par l'État, est bien illustrative du poids des pouvoirs coutumiers dans le succès ou l'échec des politiques publiques. L'intervention des autorités coutumières dans le système informel d'organisation de la production aurifère, a pour effet de garantir la continuité de cette économie très lucrative pour les populations locales et de marginaliser un État central bien souvent éloigné des intérêts des acteurs locaux. La volonté de l'État de formaliser et rationaliser le processus d'exploitation artisanale au profit d'investissements porteurs, a davantage privilégié une approche répressive que consensuelle, à même de garantir une juste répartition des ressources entre les intérêts communautaires et ceux de l'État.

\subsubsection{Les propriétaires de terre et de plantation}

Les propriétaires de terre tirent eux aussi les retombées économiques de l'orpaillage. Leur gain se fait à travers le paiement d'un ticket d'exploitation. Le coût du ticket varie selon le genre de l'exploitant et le type d'exploitation. Ainsi, les hommes et les femmes qui font le lavage simple ou la recherche de pierre doivent payer respectivement 1000 et 500 FCFA par semaine soit l'équivalent de 1,71 \$ US et $0,85 \$$ US. Quant à ceux qui font le lavage à la batée ${ }^{9}$, ils doivent payer 7000 FCFA par semaine soit l'équivalent d'environ 12 \$ US. Ces montants sont exigés par les propriétaires de terre pour l'exploitation des sites. Sur un site où la plantation de cacao d'un particulier est détruite chaque jour, le propriétaire exige une somme de 5000 FCFA par orpailleurs par semaine. Des contrôles réguliers sont faits par les propriétaires de terre pour s'assurer que toute personne exerçant sur leur terre a payé son ticket. Dans le cas échéant, l'orpailleur est contraint de se mettre en règle sous la menace d'être expulsé du site. Le non-paiement de ces droits d'exploitation entraine souvent des conflits entre les propriétaires de terre et les orpailleurs. Ces conflits aboutissent souvent à la fermeture momentanée des sites d'exploitation. Le cas du site de Bouakako est différent. Là-bas, les acheteurs d'or se sont regroupés pour acheter le site d'exploitation aux Autochtones. Le contrat d'achat monte à 3 millions de FCFA (soit 5120 \$ US) en espèce, des chaises en plastique et des bâches, plus des animaux à sacrifier. Dans tous les cas, l'enjeu économique est tel que le non-respect des termes du contrat entraine des conflits. À Bouakako, les Orpailleurs ne prennent pas le ticket d'exploitation, cependant ils sont tenus de vendre leur production aux acheteurs propriétaires du site sous peine de 
se voir expulser. La différence est qu'à défaut de payer des tickets les orpailleurs vendent leur or à un prix forfaitaire de 7500 FCFA (soit 13 \$ US) au lieu de 8500 FCFA (14,5 \$ US) ou 9000 FCFA (15,4 \$ US) sur les autres sites.

\subsubsection{Les « grands exploitants » ou les « patrons » ou les promoteurs de l'activité : les Burkinabés, les Maliens, les Guinéens}

Les promoteurs de l'activité, notamment des exploitants en général étrangers (Burkinabé, Maliens et Guinéens) disposant de moyens de production et contrôlant les circuits d'écoulement de la production vers les marchés informels de la sousrégion, ont astucieusement structuré l'activité autour des influentes autorités traditionnelles et des jeunes de la localité.

Ce sont des orpailleurs qui possèdent les facteurs de production et un fonds de roulement plus ou moins élevé. Ce capital leur permet d'engager dans leur unité de production de la main-d'œuvre. Il faut en moyenne 10 à 12 ouvriers au service de ce " patron ». Il les paie journellement à la fin de leur service. Les patrons utilisent souvent leurs enfants en vacances ou sans emploi pour les aider dans leur activité. Selon eux, utiliser les siens pour travailler sur les sites est plus bénéfique en ce sens que dans ces conditions, l'argent reste dans la famille. Nous avons identifié environ 45 grands exploitants réguliers sur les sites. La plupart d'entre eux exercent sur le site. Seulement 2 possèdent une motopompe qui les alimente en eau pour le lavage de l'or. Ces « grands orpailleurs » utilisent en général la méthode du lavage à la batée dans la recherche de l'or.

Une grande partie de cette catégorie d'acteur n'est pas novice dans l'activité. Ils ont déjà exercé ailleurs et leur ancienneté dans l'activité leur permet d'acquérir le fonds de roulement, les moyens de production et les connaissances pratiques dans le lavage du fond limoneux contenant les particules d'or. En réalité, ces grands exploitants ont commencé pour certains en tant qu'ouvriers, pour d'autres auprès de leurs parents. Dans tous les cas, ces " grands exploitants" ne sont pas venus sans avoir appris quelque part. Certains d'entre eux ont quitté les sites de Kokoumbo, Agbaou, Bouaflé; Issia... jugés presqu'épuisés. D'autres sont des anciens orpailleurs devenus planteurs de café, de cacao ou producteurs de vivriers et qui se reconvertissent à leur ancienne activité étant donné l'occasion qui se présente à eux. Ils sont de diverses nationalités. Attirés par les gains financiers dans cette activité, ils se sont rués vers la ville de Hiré.

\begin{tabular}{|l|l||}
\hline NATIONALITÉ & EFFECTIF \\
\hline \hline Ivoirienne & 8 \\
\hline \hline Malienne & 19 \\
\hline \hline Ghanéenne & 6 \\
\hline Burkinabé & 10 \\
\hline \hline & \\
Guinéenne & 2 \\
& \\
\hline
\end{tabular}

Tableau 1 - Répartition des grands orpailleurs recensés sur le site d'Assayè selon leur nationalité Source : Données d'enquête 2018

Selon ce tableau, on constate que les orpailleurs de nationalité malienne et burkinabée sont les plus nombreux. Cela s'explique en partie par leur habitude dans cette activité. En effet, l'orpaillage est une pratique très développée au Mali et au Burkina Faso. 
Depuis sept ans, Sékou, un Malien, est propriétaire d'un site minier d'or près de Sakassou, au centre de la Côte d'Ivoire. Originaire du Burkina Faso, il a d'abord dû conclure une entente avec le chef du village et le propriétaire foncier avant de commencer à exploiter le site. Pour chaque approbation, il a dû débourser 500000 FCFA (environ 860 \$ US). Il a dû négocier ensuite avec le préfet, qui a prélevé une autre commission fondée sur la production : $500 \mathrm{FCFA}(0,85$ \$ US) pour chaque gramme produit sur son site vont au chef du village et 1500 FCFA (2,55 \$ US) de plus vont au propriétaire foncier. Environ 300 mineurs travaillent sur le site d'exploitation minière d'or de Sékou. La plupart sont des migrants venus de la Guinée, du Mali et du Burkina Faso. Les mineurs doivent vendre à Sékou toute leur production. Toutefois, il arrive souvent que celui-ci n'ait pas suffisamment de liquidités pour acheter toute la production, et les mineurs peuvent être tentés de vendre ailleurs, où ils pourraient obtenir un meilleur prix. En plus des 2000 FCFA (3,40 \$ US) de taxes coutumières payés par gramme, Sékou dépense 1000 FCFA $(1,70$ \$ US) par gramme pour le mercure nécessaire au traitement de l'or. Finalement, chaque gramme lui coûte environ $18000 \mathrm{FCFA}(30,60$ \$ US), dont $15000 \mathrm{FCFA}(25,50$ \$ US) servent à payer les mineurs. Il revend le gramme 2000 FCFA (3,36 \$ US). Pour le moment, il peut acheter $1 \mathrm{~kg}$ d'or par semaine, mais s'il avait plus d'argent, il pourrait facilement en acheter 1,5 $\mathrm{kg}$ par semaine. Il explique que durant la rébellion, il y avait beaucoup d'argent et il pouvait acheter au moins $2,5 \mathrm{~kg}$ par semaine. Sékou reçoit des avances en espèces de Maliens avec qui il a établi des relations. Ils achètent l'or à Bouaké et le fondent à Bamako. Pour empêcher les mineurs de fuir avec leur production, Sékou paie trois anciens combattants des Forces nouvelles afin de sécuriser le site, ce qui lui coûte 150000 FCFA (255 \$ US) par mois. Sékou était d'abord venu en Côte d'Ivoire pour travailler dans le secteur du diamant, mais l'embargo sur les diamants a rendu le travail difficile et moins sûr, ce qui l'a poussé à réorienter son travail dans le commerce de l'or. Il ne regrette pas son choix parce qu'il est beaucoup plus facile de trouver du préfinancement pour le commerce de l'or que pour celui des diamants.

Encadré 1 - Récit de vie de Sékou Sidibé, « grand exploitant » malien

\subsubsection{Les petits exploitants}

Ils sont les plus nombreux. Leur nombre peut être estimé à plus de 1500 personnes sur l'ensemble des sites. En vérité, il est difficile d'avoir une idée exacte de leur effectif. Car chaque jour de nouvelles personnes sont intéressées par ce type d'exploitation. Ils sont plus nombreux sur les sites de Djangobo et d'Assayé. Le genre féminin est dominant dans cette catégorie d'exploitants. Ce sont des femmes au foyer, veuves, jeunes filles, commerçantes de vivriers qui s'adonnent à cette activité. Il n'est cependant pas rare de voir aussi des élèves, des jeunes sans emplois et même des hommes adultes dans cette catégorie d'exploitants. C'est parfois même toute la famille au complet qui s'engage à l'exploitation de l'or. La quasi-totalité de ces exploitants n'a pas d'autorisation d'exploitation. Ils opèrent tous dans la clandestinité (illégalité). Dans l'incapacité de se procurer les moyens de productions de masse, ils se contentent de creuser la terre à la recherche de pierres ou encore de ramasser celles rejetées par les grands exploitants. Les «Petits» exploitants sont aussi ceux qui font le lavage simple à l'aide de calebasse dans les petits barrages qu'ils construisent. Nous les qualifions de « Petits » exploitants du fait du nombre de personnes dans l'unité de production. Ils exercent très souvent seuls ou avec un membre de la famille. En les qualifiant ainsi, nous faisons allusion à la modicité de leurs moyens de production (daba, machette) et de la quantité d'or obtenu par jour. Leur rentabilité journalière s'élève à environ $1 \mathrm{~g} /$ jour très souvent moins que cela.

\subsubsection{Les ouvriers journaliers}

Ce sont des jeunes (filles et garçons) élèves, sans emploi. Ce sont aussi des demandeurs d'emploi à la société d'exploitation industrielle de l'or à Hiré (Equigold). Ils offrent leur force de travail aux " grands exploitants » à un prix de 1500 FCFA (soit 2,60 \$ US)/jour. La journée pour eux commence à $8 \mathrm{~h}$ et prend fin à $13 \mathrm{~h}$. Pour ceux qui font la journée continue, c'est-à-dire de 8 h à 16 h $30-17$ h, le salaire journalier est fixé à 2000 FCFA (soit environ 3,41 \$ US). Leur travail consiste à creuser la terre, à transporter jusqu'au bassin où se fait le lavage et enfin à laver la terre. Les tâches des ouvriers sont souvent classifiées en fonction du genre. Les hommes sont affectés aux tâches qui nécessitent beaucoup de forces physiques. Ainsi, ils s'occupent du creusage de la terre et du lavage. Les femmes, quant à elles, s'occupent du 
transport de la terre creusée et aussi à servir l'eau pour le lavage. Cependant, l'attribution de ces postes n'est pas figée. Il arrive que des femmes lavent la terre ou encore que des hommes assurent le transport de la terre jusqu'au lavage. Le travail d'ouvrier journalier dans les unités de production artisanale est très prisé par la population. Il représente $33,33 \%$ de la population sur les différents sites. Cela parce que le salaire est payé sur-le-champ juste à l'heure de la descente, sans tenir compte de la quantité d'or produite. D'ailleurs, les ouvriers ne s'occupent pas du lavage final qui permet de voir les particules d'or. Cette tâche est à l'actif du propriétaire de l'unité de production. Il le fait chez lui à la maison.

\subsubsection{Les municipalités}

De l'entretien que nous avons eu avec le Secrétaire général de la mairie d'une localité du pays qui est Hiré dans le centre-ouest, il ressort que la municipalité ne tire aucun profit de l'exploitation artisanale de l'or. Cependant, les orpailleurs nous ont informés qu'au début de leur activité, les agents de la mairie leur faisaient payer des taxes communales. Cette pratique s'est arrêtée à la suite des plaintes et à l'opposition des orpailleurs et des propriétaires de terres. Ceux-ci ont trouvé injuste que la mairie prélève des taxes sur leur propriété. Par ailleurs, nous avons remarqué que si la municipalité prétend ne pas avoir des intérêts de l'orpaillage, il n'en demeure pas moins que cette activité se fait à un certain niveau à son avantage. Cela s'explique par le fait que l'orpaillage absorbe une grande partie de la population active à Hiré. Cette activité résout donc en partie le problème grandissant du chômage à Hiré et dont les autorités municipales ne semblent pas avoir de propositions concrètes pour sa résolution.

L'orpaillage serait donc pour la municipalité un «mal nécessaire » parce qu'en même temps qu'il vide le marché de ses commerçants, il permet d'absorber la jeunesse au chômage. Du coup, même si la municipalité ne tire pas des intérêts économiques de façon directe, elle voit une partie de ses préoccupations sur le plan de l'insertion des jeunes résolue par l'orpaillage. Le problème demeure dans la durabilité de cette solution, car les gites s'appauvrissent et la dégradation causée par l'orpaillage ne supporte pas les conditions de cette pratique à long terme.

\subsection{Les caractéristiques sociodémographiques des orpailleurs}

\subsubsection{La structure par âge}

Il est ressorti de l'étude que 32474 personnes s'activent de façon directe dans l'exploitation traditionnelle de l'or dont 27444 dans l'extraction, 3814 dans le broyage et le concassage et 1216 dans l'alluvionnaire. La main-d'œuvre enregistrée dans l'activité d'extraction et du concassage/broyage est composée de 28746 hommes et de 2512 femmes. Il est noté également que $0,5 \%$ de cette main-d'œuvre est composée d'enfants de moins de 15 ans. Toutefois, la quasi-totalité des acteurs s'activant dans l'alluvionnaire est composée de femmes.

La structure par âge selon le sexe des orpailleurs de l'activité d'extraction du minerai d'or montre que les individus s'activant dans cette activité sont majoritairement jeunes. En effet, plus de 71,3\% d'entre eux sont âgés de moins de 35 ans dont $44,5 \%$ des hommes et $36 \%$ des femmes sont dans la fourchette d'âge 25 et 34 ans. Toutefois, on observe une implication dans la chaine de production, d'enfants de moins de 15 ans qui représentent $0,5 \%$ du total des actifs. La proportion des filles dans cette tranche est plus importante que celle des garçons $(3 \%$ contre $0,3 \%)$.

Les résultats montrent également que $10 \%$ des acteurs sont âgés de plus de 45 ans. Il est aussi noté que la majorité de la population active, aussi bien chez les hommes que chez les femmes, est composée de personnes qui se situent dans la fourchette d'âge 15-34 ans, 70,8 \% pour les hommes, et $29,2 \%$ pour les femmes.

La structure par âge des broyeurs et concasseurs montre que les individus s'activant dans cette activité sont majoritairement jeunes. En effet, 87,6 \% de ces individus sont âgés de moins de 35 ans dont $55,3 \%$ des hommes et $44,7 \%$ des femmes sont dans la fourchette d'âge 15 et 24 ans. Les résultats montrent également qu'aucune femme âgée de plus de 35 ans ne s'active dans le concassage ou le broyage.

\subsubsection{La situation matrimoniale}

L'analyse de la situation matrimoniale des orpailleurs montre que les mariés sont majoritaires 
(62,7\%), suivis des célibataires (36,9\%). Les divorcés, les veufs et les concubins sont les moins représentés avec des proportions respectives de $0,2 \%$, $0,1 \%$ et $0,1 \%$. Quel que soit le sexe, les orpailleurs mariés sont majoritaires; mais les plus grandes proportions se retrouvent chez les femmes avec un taux de 78,3\%. En effet, le nombre de célibataires est plus important chez les hommes que chez les femmes.

Une analyse de la situation matrimoniale des personnes évoluant dans l'activité de concassage et broyage fait ressortir que la population est majoritairement composée de célibataires. Toutefois, il est noté une différence dans cette structure selon le sexe. En effet, 59,9\% des hommes sont des célibataires, alors que la proportion des femmes mariées est de 69,7\%. L'importante proportion de célibataires enregistrée est en liaison avec leur jeunesse (plus de $55 \%$ ont moins de 25 ans).

\subsubsection{Le taux d'instruction}

Le niveau d'instruction permet de voir si l'individu a fréquenté l'école à des cycles différents ou s'il a reçu des cours d'alphabétisation.

Le niveau d'instruction des orpailleurs de l'activité d'extraction du minerai d'or indique que plus de 7 individus sur 10 n'ont fait que des études primaires. Le niveau d'études secondaires regroupe $17,9 \%$ des orpailleurs. De faibles proportions d'individus sont observées dans le niveau supérieur $(6,2 \%)$ et les personnes sans niveau d'instruction $(3,2 \%)$. Une analyse suivant le sexe révèle que la proportion des filles qui ont le niveau primaire est supérieure à celle des hommes avec respectivement $84,6 \%$ et $71,2 \%$. Cette tendance est la même pour ceux n'ayant aucun niveau d'instruction (4,6\% contre 3,0\%). Par ailleurs, les proportions des hommes qui ont le niveau secondaire et supérieur sont plus élevées que celles des femmes avec respectivement $18,8 \%$ et $6,7 \%$ contre $6,7 \%$ et $1,3 \%$.

En ce qui concerne le niveau d'instruction des broyeurs et concasseurs, notons que cette population est à majorité composée d'individus n'ayant pas été dans le système éducatif, soit environ $60,9 \%$. Une analyse comparative selon le genre montre que la proportion d'hommes (60,8\%) n'ayant pas fréquenté l'école est presque équivalente à celle des femmes $(60,9 \%)$. La proportion d'individus ayant fait des études primaires s'élève à
20,6\%. Cependant, celle des hommes est plus importante que celle des femmes $(20,8 \%$ contre 9,1\%). La proportion des individus ayant fait des études supérieures est faible $(0,6 \%)$.

\subsubsection{La nationalité}

Au cours de l'enquête, diverses nationalités ont été recensées. L'analyse de la nationalité des orpailleurs de l'activité d'extraction du minerai d'or révèle que plus de 4 individus sur 10 sont de nationalité burkinabé. Les Maliens sont fortement représentés, soit 39,6 \% des orpailleurs. Les Guinéens et les Ivoiriens suivent avec des proportions respectives de 10,3\% et $5,2 \%$. Les Sénégalais, les Ghanéens et les Mauritaniens sont faiblement représentés, soit respectivement $0,2 \%, 0,1 \%$ et $0,1 \%$. Les Togolais, Nigériens, Béninois, etc. sont les moins représentés. L'analyse de la nationalité des broyeurs et concasseurs montre que les non-Ivoiriens sont majoritaires dans cette activité avec plus de $84,2 \%$ du total des individus, alors que les Ivoiriens représentent 15,8\%. Les autres nationalités proviennent essentiellement du Mali, du Burkina Faso et de la Guinée, avec respectivement 45,6 \%, $34,7 \%$ et $3,7 \%$.

\subsubsection{L'ancienneté dans l'activité}

L'ancienneté dans l'unité de production est une information qui permet de déterminer le temps que l'individu a effectué dans l'exercice de ses fonctions. Les données sur l'ancienneté des orpailleurs de l'activité d'extraction du minerai indiquent que $42,4 \%$ des individus font moins de 3 mois dans leurs puits, ou tranchées. Par rapport à leur lieu d'exercice, environ 3 orpailleurs sur 10 ont une ancienneté comprise entre 3 et 6 mois et 20,4\% entre 9 et 12 mois. Seuls 8,1\% des individus ont entre 6 et 9 mois d'ancienneté.

Les données sur l'ancienneté des broyeurs/concasseurs dans le site d'exercice indiquent que la plus grande proportion des individus n'a pas fait plus d'une année dans le même site, soit 67,4\% des acteurs. Les résultats révèlent également que le pourcentage d'individus diminue presque avec l'accroissement du nombre d'années. Seuls 3,8\% des acteurs ont pratiqué l'activité de broyage ou de concassage durant plus de 4 ans. 


\subsection{Les impacts économiques de l'orpaillage}

L'exploitation minière est encore en phase de démarrage, sa contribution au PIB national s'élevant à seulement $0,2 \%$ environ, et son apport aux ressources intérieures de l'État étant inférieur à $1 \%$. Le secteur minier ivoirien comprend l'extraction d'or et de diamants, ainsi que les dépôts de fer, de nickel, de manganèse et de bauxite, entre autres. Selon des estimations de la Direction Générale des Mines et de la Géologie (DGMG), le secteur de l'exploitation artisanale et semi-industrielle d'or et de diamants emploie officiellement entre 5000 et 8000 personnes directement. En tenant compte du secteur minier informel et de l'emploi indirect, 100000 à 300000 personnes seraient dépendantes du secteur. Parallèlement aux opportunités d'emploi, les entreprises minières instaurent d'importantes actions socioéconomiques et culturelles en faveur des populations locales en termes d'infrastructures à caractère communautaire (construction de centres de santé, d'écoles, travaux d'électrification et/ou d'adduction d'eau potable, etc.).

\subsubsection{Production de l'orpaillage}

Les résultats de l'étude ont montré que l'activité d'orpaillage a produit en 2017 une production de 4,3 tonnes d'or, dont 4,0 tonnes pour l'orpaillage pratiqué dans l'activité d'extraction du minerai d'or et 341 kilogrammes d'or pour l'orpaillage alluvionnaire. La production en valeur de l'or est de 86, 6 milliards de FCFA dont 80,2 milliards de FCFA (soit environ 137 millions \$US) dans l'activité d'extraction et de 6,4 milliards de FCFA (soit environ 109 millions \$ US) dans l'orpaillage alluvionnaire. Les consommations intermédiaires sont évaluées à 12,7 milliards de FCFA (soit environ 22 millions \$ US). Ainsi, la valeur ajoutée dégagée par l'orpaillage est-elle de 73,9 milliards de FCFA (soit environ 126 millions \$ US).

La production annuelle de services de soutien (broyage/concassage) est estimée à 5,1 milliards de FCFA (soit environ 8,7 millions \$US). Elle est répartie entre les services de broyage (2,6 milliards de FCFA) et de ceux de concassage (2,1 milliards de FCFA). Les consommations intermédiaires sont évaluées à 1,4 milliard de FCFA. La valeur ajoutée dégagée par l'activité de broyage/concassage est estimée à 3,7 milliards de FCFA.

\subsubsection{La commercialisation de l'or: la renaissance du métier de « commerçants »}

L'or obtenu est vendu sur place. Les acheteurs viennent d'Abidjan. Certains viennent juste acheter l'or et retournent chez eux. D'autres ont trouvé judicieux de s'installer à Hiré pour acheter l'or. D'autres encore, ceux-ci résidant à Hiré, se sont transformés en acheteurs d'or pour la circonstance. Dans tous les cas, la vente est facile. Selon les orpailleurs, l'or ne peut pas rester invendu par manque d'acheteurs. C'est justement l'une des raisons qui motivent les orpailleurs. Contrairement à l'agriculture et au commerce où il arrive que les produits ou la marchandise restent invendus faute d'acheteurs, l'or lui est vendu immédiatement. La demande étant forte, les acheteurs adoptent les attitudes concurrentielles qui consistent à fournir le matériel pour réduire la roche en poudre. D'autres se sont associés pour acheter le gite de Bouakako et tout exploitant est tenu de leur vendre sa production. La vente se fait par pesée à l'aide d'une balance. Le prix du gramme d'or oscille sensiblement entre 8500 et 9000 FCFA (soit entre 14,5 et 15,4 \$ US). Le circuit utilisé par les orpailleurs est un circuit direct ce qui leur permet d'échapper aux règles en vigueur c'est-à-dire les taxes et autres frais. Cette situation crée donc une condition favorable et confortable d'exploitation et de vente de l'or à Hiré.

Dans la plupart des cas, l'or est vendu directement sur place à de petits acheteurs. On utilise des balances locales ainsi que des poids simples comme des pièces d'un FCFA et des allumettes. La plupart de ces acheteurs sont des agents qui agissent pour le compte de grands commerçants des villes. Ils font partie d'un réseau informel et international dont les ramifications s'étendent jusqu'aux grands centres de commerce (Grätz, 2002). Par l'offre d'avantages immédiats, ces commerçants en or font le lien entre la production locale et l'économie de marché globale. Ils essaient de fidéliser les orpailleurs comme clients en leur garantissant des prêts qui obligent ceux-ci à leur vendre leur production en priorité. Le besoin permanent en argent liquide oblige les orpailleurs à s'inscrire dans cette logique, renforçant ainsi le volume des activités commerciales. 
La question du préfinancement est un facteur à prendre en considération si l'on veut comprendre le commerce de l'or. En général, il y a deux ou trois intermédiaires dans la chaine entre les mineurs et les exportateurs. On pourrait décrire comme suit la chaine d'approvisionnement habituelle dans les trois pays : les mineurs travaillent pour un propriétaire de site, qui vend leur production à des petits acheteurs qui exercent leurs activités près des sites miniers. Les petits acheteurs sont habituellement préfinancés par des négociants d'or plus importants, dans les grandes villes telles Ouagadougou et Bamako. Eux-mêmes sont habituellement préfinancés par des acteurs en aval, incluant des centres d'affinage tels que les Émirats arabes unis. Partout en Afrique, le commerce artisanal de l'or est basé sur un système de préfinancement. L'argent qui finance l'or va dans la direction opposée à celle de l'or. Le système fait en sorte qu'il est difficile pour les mineurs de fonctionner au sein du système légal, même s'ils le voulaient. De l'argent venu d'ailleurs paie leur équipement et leurs indemnités de subsistance quotidiennes et, en retour, leur production est déjà prépayée et leur route d'exportation prédéterminée par les négociants dans les capitales qui offrent un préfinancement pour les opérations. Le besoin d'un haut niveau de liquidités dans le secteur fait en sorte qu'un acheteur ou un exportateur d'or n'entrepose jamais de marchandises parce qu'il n'a pas de capital fixe pour conserver l'or. Son rôle est de jouer l'intermédiaire durant la transaction et de se garder une marge entre les petits acheteurs et les acheteurs étrangers. Les acheteurs et les exportateurs établis dans les plus grandes villes travaillent presque toujours avec une équipe de petits acheteurs et ils préfinancent leurs achats aux sites miniers. Au Mali, la majorité des négociants d'or à Bamako, de même que les propriétaires des sites, sont des Maliens; au Burkina Faso, ce sont des Burkinabés; tandis qu'en Côte d'Ivoire, ils proviennent du Mali ou du Burkina Faso. C'est habituellement à ce niveau que la contrebande apparait. Des acheteurs burkinabés opèrent partout en Côte d'Ivoire particulièrement dans les principales régions d'exploitation minière telles que Bouaké, Katiola, Korogo et Doropo. Conséquemment, dans certaines régions minières comme Bouna, dans le nord de la Côte d'Ivoire, la production est vendue presque entièrement à des négociants à Ouagadougou.
Entre-temps, les acheteurs à Bouaké, au centre de la Côte d'Ivoire, préfèrent vendre leur or à de plus petits acheteurs du Mali. Ces acheteurs ne sont pas nécessairement rattachés à un site particulier, et ils achètent selon la production et selon leurs capacités financières, au nom d'un plus gros client dans les capitales. L'un d'eux, qu'on appelle «le Saoudien » à Bamako, a dit qu'il préfinance entre 30 et 40 entrepreneurs, achetant en moyenne $200 \mathrm{~kg}$ par mois, qu'il exporte ensuite à Dubaï. En plus de l'or, il achète aussi illégalement des diamants de la Guinée et de la Côte d'Ivoire. Ses exportations mensuelles — qui valent plus de 8000000 \$ US aux prix actuels — illustrent bien l'ampleur du commerce illicite dans les trois pays. Le manque de traçabilité de l'or des orpailleurs artisanaux expose le commerce légitime de l'or à une grave menace. La vulnérabilité est particulièrement aiguë à l'échelon des négociants d'or dans les capitales, le dernier maillon de la chaîne avant l'exportation à l'étranger. C'est ce qu'a démontré un négociant chevronné, qui a admis n'avoir aucune idée de l'endroit où ses acheteurs trouvent l'or qu'il exporte.

\subsubsection{Les revenus engrangés après la commercialisation : l'or, une rentabilité rapide et élevée}

Les revenus engrangés permettent d'avoir accès à tout un ensemble de biens de consommation symboliques du prestige et de la réussite sociale au niveau local. Cependant, l'orpaillage a aussi provoqué une inflation des prix des denrées alimentaires dans la plupart des zones rurales et villages comme en témoigne le secrétaire de l'association des jeunes du village de Agbaou : "Ici, on vendait le kg de viande de bouf à $1000 \mathrm{fr}$. il y a trois ans. Mais maintenant il est à 2200 comme à Abidjan. L'igname dont le tas était à 500 fr. est montée à 2000 fr. Elle est trois fois moins chère à Dabakala qu'ici.». La répartition des revenus selon la part moyenne en gramme d'or et le revenu moyen annuel d'un orpailleur est la suivante :

- le « creuseurs » gagne 4 grammes d'or par puits et 67 grammes d'or annuellement. Ils ont un revenu annuel de 1,4 million de FCFA (soit environ 2400 \$ US);

- le chercheur gagne 24 grammes d'or par puits/dama et 221 grammes annuellement. À ce titre, il faut noter que le chercheur a un revenu annuel de 4,4 millions de FCFA; 
- le financier gagne en moyenne 8 grammes d'or et a annuellement 122 grammes d'or. Toutefois, il a un revenu annuel de 2,4 millions de FCFA;

- le fonceur et le tireur ont respectivement 13 et 17 grammes d'or par puits/dama. Ils ont annuellement des revenus respectifs de 2,0 millions et 2,3 millions de FCFA;

- le responsable de puits ou le représentant peut gagner 18 grammes d'or par puits/dama et 140 grammes d'or annuellement. Ceci correspond à un revenu annuel de 2,8 millions FCFA, montrant que le responsable de puits occupe une part significative dans la répartition;

- le "propriétaire de la terre » peut obtenir par dama et annuellement les quantités respectives de 22 grammes et 129 grammes d'or. $\mathrm{Il}$ peut se retrouver annuellement avec un revenu de 2,5 millions de FCFA.

Contrairement aux activités agricoles, l'orpaillage permet aux acteurs d'avoir rapidement de l'argent. C'est ce qui d'ailleurs motive une grande partie de la population $(46,66 \%)$ qui se rue sur les sites d'orpaillage. En effet, pour chaque type ou méthode d'exploitation, l'or est recueilli sur place le même jour ou 2 jours après. Pour l'exploitation souterraine de Bouakako par exemple, la production est vendue sur place le soir même de la production. Pour les autres types d'exploitation, l'or est obtenu soit le lendemain de l'exploitation ou encore deux jours après. Le traitement final qui permet d'obtenir l'or se fait selon le gré de l'exploitation. Il y a des orpailleurs qui collectent le sable fin ou sédiment (obtenu après lavage) ou encore les roches possédant des paillettes d'or et les traitent de façon définitive en temps voulu. Selon les orpailleurs, le fait d'avoir en un temps record de l'or à vendre et par conséquent avoir de l'argent justifie leur choix pour cette activité. Par ailleurs, le témoignage des orpailleurs révèle que le sol de Hiré serait plus dense en or et que cet or aurait une masse volumique plus élevée que les autres localités telles Agbaou, Kokoumbo... C'est d'ailleurs pourquoi des orpailleurs de profession se sont déplacés de différentes régions pour se rendre sur les sites de Hiré. Le rendement journalier dépend du type d'exploitation.

\begin{tabular}{|l|c|}
\hline Type d'exploitation & Rendement journalier moyen \\
\hline \hline Lavage à la batée & $4-5 \mathrm{~g}$ \\
\hline \hline À travers les roches & $0,5-1 \mathrm{~g}$ \\
\hline \hline Lavage simple & $0,5-1 \mathrm{~g}$ \\
\hline \hline Recherche souterraine & $10-11 \mathrm{~g}$ \\
\hline
\end{tabular}

Tableau 2 - Le rendement journalier moyen selon le type d'exploitation Source : Données d'enquête 2018

Notons que le rendement n'est pas fixe. Il peut arriver des jours où l'orpailleur ne trouve pas d'or ou encore une faible quantité. D'autres jours encore, l'orpailleur peut obtenir une quantité très importante. La question de chance est donc considérée. Il faut aussi préciser les considérations métaphysiques et les pratiques religieuses qui ont cours sur les sites. De l'avis général des orpailleurs, l'or est habité par des esprits, c'est pourquoi son exploitation exige des rites et des sacrifices. Ainsi, sur le site d'Assayé, certains orpailleurs exigent qu'on se déchausse sous prétexte que la chaussure ferait fuir l'or. Mais tous les orpailleurs ne croient pas à cette pratique. Sur le site de Djangobo, le sacrifice d'un bœuf est demandé. Selon les orpailleurs, l'absence ou la non-exécution de ce sacrifice serait la cause des fréquents accidents sur ce site. À Bouakako également les propriétaires de terre demandent aux orpailleurs de fournir un bœuf plus un bouc pour un sacrifice sur le site afin de demander le pardon et la faveur des esprits pour éviter les accidents et aussi que l'or se laisse trouver. Jusqu'à ce que nous quittions le site, seul le sacrifice du bouc a été exécuté. On comprend combien l'exploitation de l'or est importante pour les orpailleurs, alors qu'ils sont même prêts à faire des sacrifices pour assurer la survie de leur activité. 


\subsection{Les impacts sociaux de l'orpaillage}

\subsubsection{Assurance maladie en miniature}

Les mineurs paient une cotisation à des leveurs de fonds pour les situations d'urgence. Les entrepreneurs miniers, les chefs d'équipe et les commerçants qui ont le mieux réussi sont supposés contribuer plus que les autres, en payant, par exemple, les soins d'un travailleur malade ou en finançant une cérémonie importante, ce qui limite leur accumulation directe et, en cela, mérite d'être souligné.

\subsubsection{Esquisse de développement communautaire}

Contrairement aux sociétés d'extraction minières qui n'ont pas encore dédommagé les populations pour l'utilisation de leurs terres, les mineurs artisanaux paient aux communautés diverses contributions en espèces ou en nature. Les mineurs les considèrent souvent comme des taxes, même si elles sont partiellement redistribuées durant les festivités ou aident à améliorer les infrastructures communautaires comme les écoles, les centres de soin, les églises, etc., qui sont ouverts à tous. Cet avantage est tout à fait significatif pour des communautés de mineurs installées à proximité, voire à l'intérieur même des villages. Mais, même dans un camp minier plus isolé, la solidarité se met en place de multiples façons.

\subsection{Les impacts environnementaux de l'orpaillage}

\subsubsection{Dégradation des sols et des terres cultivables}

La dégradation des sols par les activités d'orpaillage est le plus visible d'un point de vue environnemental. Le couvert végétal des sites est dégradé par l'action des fouilles. Les orpailleurs dégagent la végétation avant de creuser la terre. Dans cette logique, toutes les herbes et les arbres sont coupés. Ainsi, les sites d'orpaillage sont des terrains rendus nus sur des centaines de mètres. Les trous creusés par les orpailleurs, les pierres infertiles en or rejetées à la surface rendent les sites irrécupérables pour l'agriculture. Dans un premier temps, les trous rendent les sites d'accès dangereux et dans un second temps, ils compromettent l'usage de ces sites pour une activité agricole. Les champs proches des sites d'exploitation sont quotidiennement détruits par l'action des orpailleurs. La dégradation des terres cultivables a conduit à un déclin de la production de bois et de l'agriculture. Ce qui s'avère particulièrement problématique pour le droit à l'alimentation des communautés. Les activités minières et l'utilisation du cyanure pour l'extraction de l'or ont pollué les ressources en eau potable des communautés, donnant à l'eau une teinte rouge.

\subsection{Les risques liés à l'activité de l'orpaillage}

\subsubsection{Les risques sanitaires}

Les effets délétères de l'orpaillage sont nombreux. Ils sont pour la plupart liés à l'hygiène des sites d'exploitation et la poudre de poussière dégagée par la pierre pilée. Les orpailleurs les plus exposés sont ceux qui pratiquent le lavage simple, le lavage à la batée et le broyage des pierres. Dans le cas du lavage simple et le lavage à la batée, la présence des orpailleurs dans l'eau insalubre tout le temps de leur activité est un risque potentiel d'infections. Ils sont vulnérables à plusieurs maladies cutanées et sexuelles à court et à long terme. Pour ceux qui broient les pierres, ils sont exposés à des infections cérébrales. Selon le médecin de la ville, environ $35 \%$ des consultations ont un rapport direct avec l'orpaillage. Il s'agit des maladies comme la bilharziose, les infections vaginales, la fièvre typhoïde, les infections cutanées, la méningite, etc. Au-delà de ces maladies, les orpailleurs sont exposés à d'autres maladies liées à l'intoxication au mercure. Même si nous n'avons pas mené des études poussées de cause à effet, des études produites ailleurs montrent que les inconvénients sanitaires de l'orpaillage sont inquiétants (Richard, Moher, Telmer, 2014). Il est à noter que les nappes phréatiques sont également polluées par le cyanure et le mercure utilisés pour purifier le minerai. Ces contaminations entrainent des cas de paralysie, de cécité et de nombreuses fausses couches.

\subsubsection{Les risques d'accident}

Les accidents sont légion sur les différents sites d'orpaillage enquêtés. Ces accidents partent des plus bénins aux plus graves, aboutissant parfois à la mort. Les accidents les plus fréquents sont causés par des éboulements et par des particules de roche qui sautent lors du concassement du broyage des 
roches et qui entrainent le plus souvent des blessures graves chez les mineurs.

\section{Discussions}

\subsection{Transmigration des orpailleurs}

Les orpailleurs migrants établissent des campements miniers, faits de tentes, de huttes et d'appentis en paille, ou bien vivent à proximité des bassins d'exploitation dans des maisons louées ou bâties par eux-mêmes. Ces sites d'orpaillage sont dispersés régionalement et ils représentent les points d'ancrage d'un microsystème économique. Dans tous les cas, le nombre des orpailleurs se réduit de manière importante durant la saison agricole et augmente considérablement durant la saison sèche. Il peut aussi varier durant les périodes de festivités ou d'obligations religieuses. Les pluies et l'assèchement des rivières peuvent également entrainer l'arrêt provisoire de l'orpaillage. Les professionnels quittent le site en premier, si de nouveaux gisements promettant d'être plus rentables sont identifiés dans d'autres régions. Ils « transplantent » alors leur camp. Un petit nombre d'orpailleurs reste dans le camp qui se vide, et les habitants des villages avoisinants continuent généralement l'orpaillage pendant la saison sèche. Cette transmigration permanente des pionniers-orpailleurs est accompagnée d'une diffusion des normes de l'orpaillage. Elle produit de nouveaux circuits économiques et génère une prolifération des compétences, des ressources monétaires et du savoir. Les règles fondamentales sont ensuite appropriées par quelques orpailleurs autochtones.

\subsection{Les défis liés à l'orpaillage traditionnel}

En Côte d'Ivoire, les activités minières se traduisent par des risques et dangers multiples pour l'environnement, l'agriculture et le bien-être des populations locales. Les travaux d'exploration et d'exploitation minière aboutissent à des destructions massives de zones forestières et de réserves fauniques, à l'érosion des sols, à une augmentation de la production des déchets, et à la pollution de l'air et de l'eau. Les activités minières menacent également les espèces rares et endémiques et les plantes médicinales, et contribuent à chasser les animaux de la zone de déboisement.
Le secteur minier est extrêmement vulnérable aux crises politiques et à l'instabilité, et le conflit ivoirien a eu de lourdes conséquences sur le secteur à travers la destruction d'infrastructures minières, la perte d'emplois et la baisse de la productivité. De nombreuses attaques contre des mines en exploitation (Ity, Séguéla, Tortiya, etc. L'attaque de la mine d'Angovia par des bandits en 2006 a causé la mort d'un gendarme), des vols de produits miniers (or, diamants, etc.) et la destruction d'usines et d'unité de production (notamment à Ity) ont été enregistrés. En outre, de nombreux mineurs clandestins ont profité de cette période de conflit pour piller les mines industrielles accessibles et la plupart des sites d'extraction artisanale ont été abandonnés à travers le pays à cause du climat d'insécurité.

Les conséquences sociales du secteur minier en Côte d'Ivoire incluent des effets néfastes sur la santé des populations locales, une pression accrue sur les terres cultivables, des tensions entre les populations riveraines et les exploitants. Il existe un risque important de propagation d'infections telles que le VIH/SIDA, en particulier pour les populations des localités hôtes, ou de développer des affections respiratoires et ophtalmologiques, causées notamment par le soulèvement des poussières et le dégagement des odeurs issues de la lixiviation en tas du minerai traité aux cyanures par arrosage. La pollution de l'eau en raison des déchets toxiques contenant du cyanure, du sulfure d'hydrogène et d'autres éléments a causé la mort de 20 personnes durant la période de crise dans la zone sous contrôle rebelle.

La qualité informelle de l'exploitation artisanale des mines se traduit par un manque de contrôle des activités, dont l'impact peut être considérable. Depuis 2002, on observe une absence de suivi et de contrôle notable dans ce secteur, pour ce qui est de l'activité des orpailleurs. Le travail entièrement manuel dans des conditions extrêmement difficiles crée de nombreux risques d'accident. Dans les mines artisanales d'or et de diamant (Kokumbo, Séguéla et Tortiya), de nombreux exploitants clandestins ont souvent trouvé la mort dans les galeries souterraines à cause des éboulements et des glissements de terrain. Pourtant, à part des cas de maladie (paludisme, infections ORL, allergies, etc.) dont les ouvriers sont souvent victimes, aucun cas de décès dans les mines n'a encore été officiellement signalé. De plus, les mineurs vivent souvent au 
cœur des forêts dans des zones dépourvues de centre de santé ou de personnel médical expérimenté, et où ils peuvent être victimes d'attaques à main armée par des bandits et/ou des rebelles. Par ailleurs, la recherche de pépites entre les racines de certaines plantes donne lieu à une dégradation importante des terres, exacerbée par les fréquents changements de sites par les orpailleurs en perpétuelle recherche de meilleurs gisements.

L'exploitation artisanale est freinée par la vétusté de l'équipement des orpailleurs traditionnels et leur manque d'encadrement. Enfin, la Côte d'Ivoire ne possède pas assez de moyens pour effectuer la cartographie géologique et minière de son territoire. Une meilleure connaissance des potentialités minières du sol et sous-sol, grâce à une cartographie géologique, minière et géophysique, accompagnée de la mise en place de procédures fiables pour les activités minières, permettrait au secteur d'augmenter sa contribution au développement socioéconomique du pays, tout en limitant ses impacts négatifs sur l'environnement et la santé des populations.

\section{Conclusion}

Des différents constats et analyses, il est ressorti que dans la plupart des zones rurales du pays, toutes les activités économiques, sociales et environnementales sont influencées par la pratique de l'orpaillage. Cette activité a fini par changer la configuration socioéconomique et environnementale des différentes régions. En effet, les plus grands regroupements humains dans toutes les régions où cette activité existe sont les sites d'orpaillage où se côtoient plusieurs nationalités. L'orpaillage est donc en train de détrôner la « cacao-culture » de ce point de vue. Ainsi, ces différentes régions sont devenues des zones attrayantes pour les grands investisseurs miniers du monde, mais aussi pour les grands commerçants d'or de la sous-région.
L'orpaillage constitue l'un des vecteurs essentiels de la croissance de ces régions. Il contribue de façon notable au bien-être de la population.

En 2017, l'orpaillage a généré d'une part près de 73,9 milliards de FCFA (soit environ 126 millions \$ US) dans l'activité d'orpaillage et d'autre part 3,7 milliards de FCFA (soit environ 6 millions \$ US) dans celle du broyage/concassage. Malgré cette manne financière, les zones d'orpaillage artisanal restent des régions pauvres. Par ailleurs, il est constaté que les fruits de la croissance régionale sont mal redistribués. Le nombre d'Ivoiriens travaillant de façon directe dans l'orpaillage est inférieur à l'effectif des non-Ivoiriens. Seuls 4 Ivoiriens sur 10 exercent dans le sous-secteur. D'importantes sommes d'argent sont ainsi transférées à l'étranger. Aujourd'hui, l'enjeu principal reste une répartition inclusive de la croissance tirée de l'orpaillage à toute la population de la zone d'étude.

Dans un autre volet, la forte croissance démographique, conjuguée à la forte pression sur les ressources environnementales, conduira de façon inexorable à la rupture de l'équilibre naturel du milieu si la tendance n'est pas inversée. Les activités minières ont des effets directs sur le renchérissement de la vie dans la zone d'étude. L'orpaillage devient de plus en plus mécanisé avec l'utilisation de détecteurs de métaux, de concasseurs, broyeurs motorisés et de produits chimiques dangereux. Une meilleure compréhension et une plus grande collaboration entre les différents partenaires seraient souhaitables. La recherche d'une prospérité mutuelle entre les populations, les minières et les différentes autorités gouvernementales seraient bénéfiques à tous et contribueraient à enrayer les activités illégitimes auxquelles s'adonnent ceux qui se sentent laissés pour compte dans l'accès à l'enrichissement collectif.

\section{NOTES}

1 Banque Mondiale, juin 2013, Côte d'Ivoire - Zone industrielles : Besoins et Perspectives, Rapport Abidjan; ONUDI, octobre 2012, Nouvelle Politique Industrielle de la République de Côte d'Ivoire. Phase I : Diagnostic du secteur industriel et du cadre institutionnel.

2 Ville située à l'ouest du pays aux environs de la frontière avec le Liberia

3 Village situé dans le département de Hiré

4 Ville située au sud-ouest de la Côte d'Ivoire

5 Village situé au nord de la Côte d'Ivoire dans la sous-préfecture de Tengrela.

6 Ville située dans la banlieue d'Abidjan

7 Ministère de l'Industrie et des Mines (année). Quel document? Repéré à www.industrie.gouv.ci. 
8 Bien que les marchés de l'extraction minière illégale continuent à évoluer.

9 La batée est un instrument conique en forme de chapeau chinois. C’est un moyen très efficace pour découvrir des paillettes et des pépites en milieu aurifère. L'avantage de la batée réside dans sa faculté à être transporté partout. Le chercheur d'or se rendant parfois dans des zones reculées, préférera d'abord utiliser la batée avant d'apporter sur le terrain toutes les grandes pièces d'équipements. Généralement, si les premiers essais à la batée sont positifs et qu'on découvre plusieurs échantillons de grandes valeurs, on amènera à cet endroit un équipement de production plus important pour travailler le sol et ainsi trouver davantage d'or. L'utilisation de la batée est assez basique et simple à comprendre. Par exemple lorsqu'il s'agit de l'extraction de l'or dans une rivière et lorsque l'on veut utiliser le lavage à la batée, il faut dans un premier temps comprendre comment la rivière arrive à concentrer l'or au même endroit, quels sont les mouvements que la rivière impose à l'or pour qu'il se dépose à des endroits précis. L'orpailleur place ainsi dans la batée un tas de roches, cailloux, sables qu'il aura prélevés dans l'eau ou à proximité de celle-ci. Il secoue la batée dans un mouvement de gauche à droite sous l'eau pour provoquer l'or. Ce qui est plus léger, et donc sans valeur, sera remonté et ensuite éjecté de la batée par le mouvement de gauche à droite. Le processus d'agitation et de balayage sera répété jusqu'à ce qu'il ne reste que les matériaux les plus lourds dans la batée, à savoir, l'or et le sable noire. Sur le terrain, chaque orpailleur a son propre style pour secouer, agiter, balayer avec la batée. Chaque orpailleur développe sa technique pour obtenir les meilleurs résultats.

\section{RÉFÉRENCES}

Conseil de Sécurité des Nations Unies (2014). Rapport final du Groupe d'experts sur la Cote d'Ivoire établi en application du paragraphe 27 de la résolution 2153 (2014) du Conseil de sécurité. Repéré à http:/ /www.reseau-rafal.org/sites/reseaurafal.org/files/ document/externes/ONU \%20Rapport \%20experts \%20Cote \%20d \%27Ivoire \%20avril \%2015.pdf.

Ford, T. (10 février 2014). Ivory Coast joins the African gold rush - but it's no quick fix for the economy. The Guardian. Repéré à www.theguardian.com/global-development/poverty-matters/2014/feb/10/ivory-coast-joinsafrica-gold-rusheconomy

Grätz, T. (2002). Gold Mining Communities in Northern Benin as Semi-Autonomous Social Fields. Halle, Saale: Max Planck Institute for Social Anthropology.

Hatcher, P. (2002). La troisième génération des codes miniers en Afrique. Paris, France: Hachette.

Hunter, M. (2017). The artisanal and small-scale gold mining sector, étude de cas pour l'OCDE / la BAD, Flux financiers illicites - l'économie du commerce illicite en Afrique de l'Ouest. Paris, France: OCDE.

Jasmamie, C. (juillet 2014). Randgold hoping for major discovery in Ivory Coast. Repéré à Mining.com

Leblanc, J. (Juin 2005). Les déplacements de populations dus à des projets miniers en Afrique subsaharienne : Contraintes structurelles à la prévention des risques d'appauvrissement. Paris, France: Points.

Minerals Yearbook, (2012). Côte d'Ivoire Advance Release. U.S. ecological Survey. Repéré à minerals.usgs.gov/ minerals/pubs/country/2012/myb3-2012-iv.pdf.

Nash, J. (1993). We eat the Mines and the Mines eat us. Dependency and Exploitation in Bolivian Tin Mines. New York, US: Columbia University Press.

Niangoran-Bouah, G. (1978). Idéologie de l'or chez les Akan de Côte d'Ivoire et du Ghana. Journal des africanistes. 48(1), 127-140. Repéré à http://www.persee.fr/doc/jafr_0399-0346_1978_num_48_1_1808

Nowak, M. (2006). Enjeux des nouvelles règlementations minières en Afrique. New York, US: Gama.

ONUDC (2011). Criminalité organisée et instabilité en Afrique centrale : une évaluation des menaces. Vienne, AT: Nations Unies.

Ribier-Tourniaire, L. (Janvier 2012). Impacts des activités minières sur les ressources en eau en Afrique de l'Ouest: Cas des mines auriferes, AgroParisTech-ENGREF-CCRE CEDEAO

Schneider, K. (1990). Das Gold der Lobi. Aspekte historischer und ethnologischer interpretation. Paideuma, 36, $277-290$.

Richard M., Moher P., et Telmer K. (2014). Problèmes de santé liés à l'orpaillage et à l'exploitation minière artisanale : Formation pour professionnels de la santé. Repéré à http://www.artisanalgold.org/wordpress/wp-content/uploads/2017/01/Health_ Training_Oct2014_version1.0_fr_lowQ.pdf

Taussig, M. (1980). The Devil and Commodity Fetishism in South America. Chapel Hill, US: University of North Carolina Press. 